\title{
Economic load dispatch with the proposed GA algorithm for large scale system
}

\author{
Hamed Aliyari, ${ }^{1,}$, Reza Effatnejad ${ }^{2}$, Ardavan Areyaei ${ }^{1}$ \\ ${ }^{1}$ Electrical Engineering Department, Science and Research Alborz branch, Islamic Azad University, Alborz, Iran \\ ${ }^{2}$ Electrical Engineering Department, Karaj branch-Islamic Azad University, Alborz, Iran \\ Email address: \\ hamedaliyary@gmail.com (H. Aliyari), Rezaeffatnejad@yahoo.com (R. Effatnejad), ardavan.aryaei@gmail.com (A. Areyaei)
}

\section{To cite this article:}

Hamed Aliyari, Reza Effatnejad, Ardavan Areyaei. Economic Load Dispatch with the Proposed GA Algorithm for Large Scale System. Journal of Energy and Natural Resources. Vol. 3, No. 1, 2014, pp. 1-5. doi: 10.11648/j.jenr.20140301.11

\begin{abstract}
Economic load dispatch (ELD) have been applied to obtain optimal fuel cost of generating units. Genetic Algorithm (GA) is a global search technique based on principles inspired from the genetic and evolution mechanism observed in natural biological systems. This paper presents a novel stochastic Genetic Algorithm approach to solve the Economic Load Dispatch problem considering various generator constraints and also conserves an acceptable system performance in terms of limits on generator real and reactive power outputs bus voltages, shunt capacitors/reactors, transformers tap-setting and power flow of transmission lines. The ELD problem in a power system is to determine the optimal combination of power outputs for all generating units which will minimize the total fuel cost while satisfying all practical constraints. To show its efficiency and effectiveness, the proposed GA algorithm is applied to some types of ED problems containing non-smooth cost functions of 13 and 40 generating units systems (large scale systems). The experimental results show that the proposed GA approach is comparatively capable of obtaining higher quality solution.
\end{abstract}

Keywords: Non-Smooth Cost Functions, Genetic Algorithm, Economic Dispatch, GA, IEEE Tests Systems

\section{Introduction}

Conventionally, economic load dispatch problem allocates loads to plants at minimum cost while meeting the constraints. It is an optimization problem which minimizes the total fuel cost of all committed plants while meeting the demand and losses. The optimal power system operation is achieved when both the objectives of power systems i.e. cost of generation and system transmission losses simultaneously attain their minimum values. Economic load dispatch reflects the optimal electrical output of generation facilities, to fulfill the system load demand, at the lowest possible cost, while providing power in a robust and reliable way. Economic load dispatch problem is one of the fundamental matters in power system operation. In essence, it is an optimization problem and its main objective is to cut-down the total generation cost, without breaching any constraints [1]. Preceding efforts on solving ELD problems have made use of various mathematical programming and optimization techniques [2]. Numerous techniques have been established to help solve the ELD problems, such as Particle Swarm Optimization [3], Artificial Bee Colony Algorithm [4], Genetic Algorithm [5], Pattern Search Algorithm [6], Neural
Networks [7], Evolutionary Programming [8], and Harmony Search Algorithm [9]. Each of the employed techniques may have some advantage and disadvantages. For instance, Particle Swarm Optimization (PSO) is well recognized for its capability to permit each particle to maintain a memory of the best solution it has discovered in the particle's neighborhood swarm. Furthermore, PSO is easy to device, and effective [10]. However, the algorithm might experience inequality constraints difficulties. Recently, Ant Colony Optimization (ACO)[11] has turn into a candidate for many optimization applications [12] such as the combinatorial optimization travelling salesman problem (TSP), quadratic assignment problem (QAP), and optimal design and scheduling problem of thermal units [13].

This paper presents an innovative approach based on Ant Colony Algorithm was chosen for solving the load-flow problem.

\section{Economic Load Dispatch}

The objective of Economic Load Dispatch is to minimize the operating cost of each generating unit in the system. Thus, an optimal generated output can be acquired from the 
solution. Economic Load Dispatch can be calculated by using the following equations[15-19]

$$
\text { optimum } \cos t=\sum_{i}^{N g} F i(P i)
$$

Where cost is the operating cost of power system and the objective function is to minimize the cost. $N g$ is the number of units. $F i\left(P_{i}\right)$ is the cost function and $P i$ is the power output of the unit $i . F i\left(P_{i}\right)$ is usually approximated by a quadratic function of its power output $P i$ as:

$$
F i(P i)=a_{i}+b_{i} P_{i}^{2}+b_{i} P_{i}+c_{i}
$$

Where $a i, b i$, and $c i$ are the cost coefficients of unit $i$. The above equation is subjected to both the equality and inequality constraint as follow:

Real power balance constraint is given by:

$$
\sum_{i}^{N g} F i(P i)=P_{D}+P_{L}
$$

Real power generation limit is given by:

$$
P_{i \min } \leq P_{i} \leq P_{i \max }
$$

Where $P_{D}$ is the total load demand in MW, $P_{L}$ is the total transmission loss of the system in MW; Pimin and Pimax are the minimum and maximum generation limit of $P i$. Next, The search of the optimal control vector is performed using into account the real power flow equation which present the system transmission losses $(P L)$. These losses can be approximated in terms of $B$-coefficients as [20]:

$$
P f=\left(1-\frac{\partial P_{L}}{\partial P_{g}}\right)^{-1}
$$

These losses are represented as a penalty vector [21-24]given by:

$$
P_{L}=\sum_{i}^{N} \sum_{j}^{N} P_{i} B_{i j} P_{j}
$$

The transmission loss of a power System $P L$ can be calculated by the $B$-Coefficients method [25] and given by:

$$
P_{L}=\sum_{i}^{N} \sum_{j}^{N} P_{i} B P_{j}+\sum_{i}^{N} B_{o i}+B_{o o}
$$

Where $B$ is an $n g \times n g$ coefficients matrix, $B 0$ is an $n g$-dimensional coefficient column vector and $B 00$ is a coefficient.

\section{Genetic Algorithm}

\subsection{Basic Principle of Genetic Algorithm}

Genetic Algorithms (GA)[26,19] are direct, parallel, stochastic methods for global search and optimization, which imitate the evolution of the living beings, described by Charles Darwin. GA is part of the group of Evolutionary Algorithms (EA). The evolutionary algorithms use the three main principles of the natural evolution: reproduction, natural selection and diversity of the species, maintained by the differences of each generation with the previous. Genetic Algorithms works with a set of individuals, representing possible solutions of the task. The selection principle is applied by means of a criterion, giving an evaluation for the individual with respect to the desired solution. The best-suited individuals create the next generation.

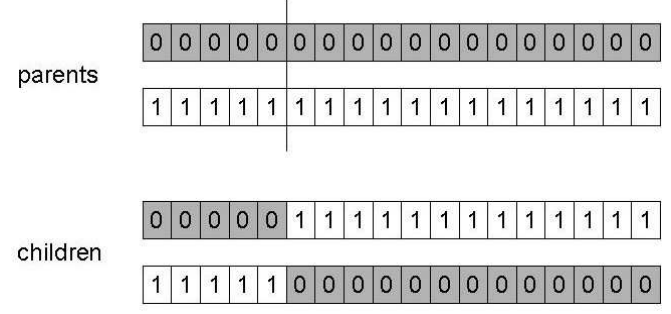

Fig 1. Behavior of Genetic Algorithm.

\subsection{Genetic Algorithm with Arithmetic Crossover}

In order to formulate the algorithm for economic/environmental ED problem, let the chromosome of the $\mathrm{k}$-th individual $\mathrm{Ck}$ be defined as follows:

$$
c_{k}=\left[P_{k 1}, P_{k 2}, \ldots, P_{k n}\right]
$$

Where

$\mathrm{k}: 1,2, \ldots$, popsize

$\mathrm{n}: 1,2 \ldots$. number_of_gene

popsize means population size, number_of_gene is the number of unit in our experiment

Pki is the generation power of the $\mathrm{n}$-th unit at k-th chromosome

Reproduction involves creation of new offspring from the mating of two selected parents or mating pairs. It is thought that the crossover operator is mainly responsible for the global search property of the GA. We used an arithmetic crossover operator that defines a linear combination of two chromosomes [23]. Two chromosomes, selected randomly for crossover, $C_{i}^{\text {gen }}$ and $C_{j}^{\text {gen }}$ may produce two offspring, $C_{i}^{g e n+1}$ and $C_{j}^{\text {gen+1 }}$, which is a linear combination of their parents i.e.,

$$
\begin{aligned}
C_{i}^{g e n+1} & =a \cdot C_{i}^{g e n}+(1-a) C_{j}^{g e n} \\
C_{j}^{g e n+1} & =a \cdot C_{j}^{g e n}+(1-a) C_{i}^{g e n}
\end{aligned}
$$


where

$C_{i}^{g e n}:$ an individual from the old generation

$C_{i}^{g e n+1}:$ an individual from new generation

a : is the weight which governs dominant individual in reproduction and it is between 0 and 1

The mutation operator is used to inject new genetic material into the population and it is applied to each new structure individually. A given mutation involves randomly altering each gene with a small probability. We generate a random real value which makes a random change in the $\mathrm{m}$-th element selected randomly of the chromosome.

\subsection{Proposed GA Algorithm for Solving ED Problem}

In this study, an innovative approach based on Genetic Algorithm was chosen for solving the load-flow problem, i.e. the two groups of genes are pretended in a parallel mood, in a way that one group moves in the values decreasing direction and the other one moves in the values increasing direction. After giving the primary values, the two groups converge to the minimum answer found.

The initialization method that is utilized in the following algorithm is an innovative method. The increasing primary amount is chosen in a way that at first, all the units should take their minimum amount and after that, we act according to the conditions 3 and 4 and the following algorithm:

A. A gene is chosen.

B. According to the probability function of one of the units, one of them is selected. Then the constant value (defined according to the accuracy and cpu time of the program, which can change based on the type of the operation), is added in accordance to the primary value that was defined for it previously. The amount of constant value should be selected in a way that it increases the power value of each unit, because each unit has already got its minimum power value. If this action does not violate condition " 4 " and that the amount of $\sum_{i}^{N g} F(P l)$ is less than $P_{D}$, then adding the constant value is accepted and proceeding to the level " $\mathrm{C}$ " is appproved, otherwise the constant value is not acceptable and this level should be repeated.

C. If the condition " 3 " is not violated, then proceeding to the next level is approved, otherwise going back to the level " $\mathrm{B}$ " is mandatory.

D. The primary amounting is done.

E. Chose another gene, i.e. go to level "A". (The number of genes is determined at first.)

We should do the same guess algorithm in a decreasing way, in which we give the maximum amount of units to them and we do the same steps in the decreasing route.

In order to get to the optimum result in accordance to the guess algorithm, in the part that we are getting the best results, the more the number of these results (genes), the more the accuracy and the less the speed of running the
code(CPU time), so the existing results give the prices of each unit.

Actually a large amount of data is obtained at the end of the process. We have to get to the most optimum level within the data, by means of GA algorithm based on arithmetic crossover and standard mutation separately.

Based on this method, the optimization was applied in 13 units [27], 40 units [28] systems (assuming power losses $P_{2}$ to be zero). It should be mentioned that in all cases, constraints such as speed and the forbidden work zones of each generator is considered.

\section{Simulation Results}

To assess the feasibility of the PSO approach, the studies of ED were compared with many optimization methods such as GA, TS, PSO, and ACO, implemented in MATLAB (7.6.0.6324(2008a) version). These programs were run on a Pentium Dual core, $2.5 \mathrm{GHz}$ personal microcomputer with 3 GB RAM under Windows XP. In each case study, 100 independent runs are carried out for each optimization method. In addition, 100 different initial trial solutions are used for each method.

The GPSO is applied to two ED problems with 13 and 40 generating units. The input data for 13 generating units system are given in [27] with 2520MW load demand. Also, the input data for 40 generating units system are given in [28] with $10,500 \mathrm{MW}$ load demand. The global solutions for these systems are not discovered yet. The best local solutions reported until now for 13 and 40 generating units are $24,169.92 \$ / \mathrm{h}$ [29] and 121,741.98 \$/h [30], respectively. After performing 100 trials, the best results for ${ }_{\mathbb{P}_{\mathrm{S}}}$, in the 13 units system and 40 units system, in order for the best answer to be found, are shown in the Tables 1 and 2 respectively.

Table 1. Best result obtained by proposed GA for 13-unit system.

\begin{tabular}{llll}
\hline unit & Pi(min) MW & Pi(max) MW & Pi MW \\
\hline 1 & 0 & 680 & 582 \\
2 & 0 & 360 & 307 \\
3 & 0 & 360 & 304 \\
4 & 60 & 180 & 150 \\
5 & 60 & 180 & 152 \\
6 & 60 & 180 & 160 \\
7 & 60 & 180 & 170 \\
8 & 60 & 180 & 151 \\
9 & 60 & 180 & 145 \\
10 & 40 & 120 & 91 \\
11 & 40 & 120 & 88 \\
12 & 55 & 120 & 112 \\
13 & 55 & 120 & 108 \\
& Total power output & $(\mathrm{MW})$ & 2521.05807120690 \\
& Total generation cost & $(\$ / \mathrm{h})$ & 24167 \\
\hline
\end{tabular}


Table 2. Best result obtained by proposed GA for 40-unit system.

\begin{tabular}{|c|c|c|c|}
\hline unit & Pi(min) MW & Pi(max) MW & Pi MW \\
\hline 1 & 36 & 114 & 102.998723543689 \\
\hline 2 & 36 & 114 & 48.8339865534452 \\
\hline 3 & 60 & 120 & 81.9858291219816 \\
\hline 4 & 80 & 190 & 184.910084237253 \\
\hline 5 & 47 & 97 & 94.2352602174419 \\
\hline 6 & 68 & 140 & 105.422756354223 \\
\hline 7 & 110 & 300 & 276.389659153576 \\
\hline 8 & 135 & 300 & 270.466620780899 \\
\hline 9 & 135 & 300 & 298.060182246735 \\
\hline 10 & 130 & 300 & 147 \\
\hline 11 & 94 & 375 & 237.691701220395 \\
\hline 12 & 94 & 375 & 206.462568526111 \\
\hline 13 & 125 & 500 & 284.649567876062 \\
\hline 14 & 125 & 500 & 299.585612037311 \\
\hline 15 & 125 & 500 & 337.915510467145 \\
\hline 16 & 125 & 500 & 276.073863005347 \\
\hline 17 & 220 & 500 & 477.22822388695 \\
\hline 18 & 220 & 500 & 498.847725762535 \\
\hline 19 & 242 & 550 & 541.67218476475 \\
\hline 20 & 242 & 550 & 420.642175667156 \\
\hline 21 & 254 & 550 & 512.202032512945 \\
\hline 22 & 254 & 550 & 530.777820055416 \\
\hline 23 & 254 & 550 & 541.468907284464 \\
\hline 24 & 254 & 550 & 521.954612690208 \\
\hline 25 & 254 & 550 & 550 \\
\hline 26 & 254 & 550 & 549.808717554535 \\
\hline 27 & 10 & 150 & 18.4444706057755 \\
\hline 28 & 10 & 150 & 17.5935208491752 \\
\hline 29 & 10 & 150 & 12.786627396382 \\
\hline 30 & 47 & 97 & 92 \\
\hline 31 & 60 & 190 & 190 \\
\hline 32 & 60 & 190 & 176.788840003834 \\
\hline 33 & 60 & 190 & 186.052294384747 \\
\hline 34 & 90 & 200 & 200 \\
\hline 35 & 90 & 200 & 190.129452886447 \\
\hline 36 & 90 & 200 & 200 \\
\hline 37 & 25 & 110 & 102.62394666683 \\
\hline 38 & 25 & 110 & 63.3481518226166 \\
\hline 39 & 25 & 110 & 108.692272455022 \\
\hline \multirow[t]{3}{*}{40} & 242 & 550 & 543.705916841164 \\
\hline & Total power output & (MW) & 10499.45 \\
\hline & $\begin{array}{l}\text { Total generation } \\
\text { cost }\end{array}$ & $(\$ / h)$ & 121168.9255 \\
\hline
\end{tabular}

In the Tables 3 and 4, there is a brief comparison between our suggested method and some other innovating methods proposed by other researchers and some standard methods based on the smart algorithms(that we discussed above) which are the results of other researchers.
Table 3. Convergence results for 13-unit system.

\begin{tabular}{ll}
\hline Method & Best Cost \\
\hline Load demand & $2520 \mathrm{MW}$ \\
Propose GA & 24167 \\
{$[31]$} & 24169.89 \\
GA & 24186.02 \\
PSO & 24171.70 \\
ACO & 24174.39 \\
TS & 24180.31 \\
\hline
\end{tabular}

Table 4. Convergence results for 40-unit system.

\begin{tabular}{ll}
\hline Method & Best Cost \\
\hline Load demand & $10500 \mathrm{MW}$ \\
Propose GA & 121168.9255 \\
{$[31]$} & 121532.41 \\
GA & 121996.40 \\
PSO & 121800.13 \\
ACO & 121811.37 \\
TS & 122288.38 \\
\hline
\end{tabular}

\section{Conclusions}

The problem of economic load dispatch with non-smooth cost functions has been investigated in this paper. We used GA algorithm with arithmetic crossover and standard mutation for arrived at this goal. A comparison analysis has been done for different intelligent techniques with respect to the total minimum generation cost that shown at the table 3 and the table 4 . The present algorithm is able to solve the economic dispatch problem under partially deregulated environment. The performance of the developed algorithm has been demonstrated on an 13 units systems and 40 units systems test system.

According to the results of this research, and the comparison between other researches results, and the significant difference between them, it is obvious that this method can be a proper method for operating gigantic power systems.

\section{References}

[1] S. M. V. Pandian and K. Thanushkodi, "Solving Economic Load Dispatch Problem Considering Transmission Losses by Hybrid EPEPSO Algorithm for Solving Both Smooth and Non-Smooth Cost Function," International Journal of Computer and Electrical Engineering, vol. 2, 2010.

[2] A. J. Wood and B.F. Wollenberg, "Power Generation, Operation and Control", New York: John Willey \& Sons, Inc., 1984

[3] S. M. V. Pandian and K. Thanushkodi, "Solving Economic Load Dispatch Problem Considering Transmission Losses by Hybrid EPEPSO Algorithm for Solving Both Smooth and Non-Smooth Cost Function," International Journal of Computer and Electrical Engineering, vol. 2, 2010.

[4] S. Hemamalini and S. P. Simon, "Economic Load Dispatch With Valve-Point Effect Using Artificial Bee Colony Algorithm," presented at XXXII National Systems Conference, 2008. 
[5] Y. Labbi and D. B. Attous, "A Hybrid GA-PS Method to Solve The Economic Load Dispatch Problem," Journal of Theoritical and Applied Information Technology, 2005.

[6] Al-Sumait, J. S., Sykulski, J. K. and Al-Othman, A. K. (2008) 'Solution of Different Types of Economic Load Dispatch Problems Using a Pattern Search Method', Electric Power Components and Systems, 36:3, $250-265$

[7] A. Y. Abdelaziz, S. F. Mehkhamer, M. Z. Kamh, and M. A. L. Badr, "A Hybrid Hopfield Neural Network - Quadratic Programming Approach for Dynamic Economic Dispatch Problem," 2008.

[8] H. T. Yang, P.C. Yang, and C. L. Huang, "Evolutionary Programming Based Economic Dispatch for Unit with Non-Smooth Fuel Cost Functions," IEEE Trans. Power System, vol. 11, no 1, pp. 112-118, Feb 1996

[9] R. Arul, D. G. Ravi, and D. S. Velusami, "Non-Convex Economic Dispatch with Heuristic Load Patterns Using Harmony Search Algorithm," international Journal of Computer Applications, vol. 16.

[10] B. H. Chowdhury and S. Rahman, "A Review of Recent Advances in Economic Dispatch," IEEE Trans. Power System, vol. 5, no. 4, pp. 1248-1259, Nov 1990.

[11] R. Effatnejad , H.aliyari, H.Tadayyoni, A.Abdollahshirazi, "Novel Optimization Based On The Ant Colony For Economic Dispatch" ,International Journal on Technical and Physical Problems of Engineering (IJTPE); Iss. 15, Vol. 5, No. 2, Jun. 2013

[12] N. H. F. I. Ismail Musirin, Mohd Rozely Kalil, MUhammad Khayat Idris, Titik Khawa Abdul Rahman, Mohd Rafi Adzman, "Ant Colony Optimization (ACO) Technique In Economic Load Dispatch," in Inrternational MultiConference of Engineers and Computer Scientist 2008, Hong Kong, 2008, p. 6.

[13] D. Nualhong, et al., "Diversity Control Approach to Ant Colony Optimization for Unit Commitment Problem," in TENCON 2004. 2004 IEEE Region 10 Conference, 2004, pp. 488-491 Vol. 3

[14] P . SUREKHA, S.SUMATHI, "Solving Economic Load Dispatch problems using Differential Evolution with Opposition Based Learning“,WSEAS TRANSACTIONS on INFORMATION SCIENCE and APPLICATIONS, Issue 1, Volume 9, January 2012

[15] F. N. Lee and A. M. Breipohl, "Reserve constrained economic dispatch with prohibited operating zones," IEEE Trans. on Power Syst., vol. 8, no. 1, pp. 246-254, 1993.

[16] J. Y. Fan and J. D. McDonald, “A practical approach to real time economic dispatch considering unit's prohibited operating zones," IEEE Trans. on Power Syst., vol. 9, no. 4, pp. 1737-1743, 1994.

[17] B. H. Chowdhury and S. Rahman , "A review of Recent Advances in Economic Dispatch", IEEE Transactions on Power Systems, November 1990, Vol.5, No. 4, pp. $1248-1259$
[18] Y. S. Haruna, Comparison of Economic Load Dispatch Using Genetic Algorithm and Other Optimization Methods, M. Eng. Degree Thesis, A. T. B. University, Bauchi, Nigeria, 2003.

[19] A. G. Bakistzis, P. N. Biskas, C. E. Zoumas, and V. Petridis, "Optimal power flow by enhanced genetic algorithm, "IEEE Trans. Power Systems, vol. 17, no. 2, pp. 229-236, May 2002.

[20] H.T. Yang, P.C. Yang, C.L. Huang,” Evolution programming based economic dispatch for units with non-smooth fuel cost functions“, IEEE Trans Power Syst 1996;11(1):112-8.

[21] C. Chen, "Non-convex economic dispatch: a direct search approach" ,Energ Conver Manage 2007;48:219-25.

[22] L.S. Coelho, V.C. Mariani, "Combining of chaotic differential evolution and quadratic programming for economic dispatch optimisation with valve-point effect", IEEE Trans Power Syst 2006;21(2):989-96.

[23] O. Abedinia, N. Amjady, K. Kiani, H.A. Shayanfar, A. Ghasemi, "MULTIOBJECTIVE ENVIRONMENTAL AND ECONOMIC DISPATCH USING IMPERIALIST COMPETITIVE ALGORITHM", International Journal on Technical and Physical Problems of Engineering (IJTPE), Iss. 11, Vol. 4, No. 2, Jun. 2012.

[24] H. Shayeghi, A. Ghasemi, "MOABC ALGORITHM FOR ECONOMIC/ENVIRONMENTAL LOAD DISPATCH SOLUTION", International Journal on Technical and Physical Problems of Engineering (IJTPE), Iss. 13, Vol. 4, No. 4, Dec. 2012

[25] S. K. Wang, J. P. Chiou, C. W. Liu, " non -smooth/non-convex economic dispatch by a novel hybrid differential evolution algorithm" ,The Institution of Engineering and Technology, 1, (5), pp. 793-803, 2007

[26] D. C. Waters and G. B. Sheble, "Genetic algorithm solution of economic dispatch with valve point loading," IEEE Trans. PWRS, vol. 8, pp. 1325-1332, 1993.

[27] N. Sinha, R. Chakrabarti, P.K Chattopadhyay," Evolutionary programming techniques for economic load dispatch",IEEE Trans Evolut Comput 2003;7(1):83-94.

[28] H.T. Yang, P.C. Yang, C.L. Huang,” Evolution programming based economic dispatch for units with non-smooth fuel cost functions“, IEEE Trans Power Syst 1996;11(1):112-8.

[29] C. Chen, "Non-convex economic dispatch: a direct search approach”, Energ Conver Manage 2007;48:219-25.

[30] L.S. Coelho, V.C. Mariani, "Combining of chaotic differential evolution and quadratic programming for economic dispatch optimisation with valve-point effect", IEEE Trans Power Syst 2006; 21(2):989-96.

[31] S. K. Wang, J. P. Chiou, C. W. Liu," non -smooth/non-convex economic dispatch by a novel hybrid differential evolution algorithm", The Institution of Engineering and Technology, 1, (5), pp. 793-803, 2007 